\title{
A Suggestion for Analysis of Unexpected Obstacles in Embedded System
}

\author{
Yasufumi Shinyashiki ${ }^{1}$, Toshiro Mise ${ }^{1}$, Masaaki Hashimoto ${ }^{2}$, Keiichi Katamine $^{2}$, \\ Naoyasu Ubayashi ${ }^{2}$, and Takako Nakatani ${ }^{3}$ \\ ${ }^{1}$ Matsushita Electric Works, Ltd., 1048 Kadoma, Kadoma, Osaka, Japan \\ \{yasufumi, tmise\} @mail.mew.co.jp \\ ${ }^{2}$ Kyusyu Institute of Technology, 680-4 Kawazu, Iizuka, Fukuoka, Japan \\ \{hasimoto@ai, katamine@ci, ubayashi@ai\}.kyutech.ac.jp \\ ${ }^{3}$ University of Tsukuba \\ nakatani@gssm.otsuka.tsukuba.ac.jp
}

\begin{abstract}
In order to improve the quality of embedded software by finding obstacles unanticipated by software specifications, this paper describes a requirement analysis method based on an Information Flow Diagram and experiments using this method, with discussion. Recently, embedded software has become more large-scale and complicated while the development cycle of the software has typically been shortened. This industry trend compromises the quality of embedded software. In order to improve the quality of the software, unexpected obstacles must be carefully analyzed in the specification and design phases. We propose a diagram-based analysis method to answer this need, and have performed an experiment using the analysis method with an actual product. The results of the experiment made it clear that 1) Novice engineers can construct an Information Flow Diagram under the lead of expert engineers; and 2) The methods by which experts lead novice engineers in the analysis of unexpected obstacles require further refinement.
\end{abstract}

\section{Introduction}

Embedded systems like electrical household appliances are used by various kinds of users in various kinds of environments [1,2]. The operations of these systems must be secure and usable over a long period. For this reason, more than $70 \%$ of embedded software is assigned to functions that handle exceptional conditions such as device faults, communication errors, signal noise, operator errors, and bad influences from the environment. Recently, embedded software has become more large-scale and complicated [3], while the development cycle of the software has typically been shortened. Therefore, it has become more difficult for engineers to anticipate exceptional conditions without accidentally leaving out specifications in the design phase of the software development. Furthermore, such work of specification requires knowledge of devices, users, and environments, as well as the software itself [4]. Therefore, engineers sometimes fail to specify certain exceptional conditions. In fact, such specification oversights cause product failure and the delay of development as software must be redesigned. Therefore, if the exceptional conditions are made clear in the specification and design phases, we can expect better quality and productivity 
in embedded software. In this paper, we define "expected specifications" and "unexpected obstacle specifications" as follows: The former specifies system behaviors usually described in the software operation manual, and explicitly defined at the start of the system architecture design. The latter concerns any deviations from the behaviors determined by the former. The deviations are as follows: the wearingout and inherent flaws of system hardware, improper operation and overload caused by system users, temperature and radio noise in the system environment, and so on. Generally, only expert engineers can anticipate unexpected obstacles with few oversights. Therefore, we studied a specification method for finding unexpected obstacles by formalizing the method that is tacitly used by experts in embedded software development $[5,6,7,8,9]$. One of the experts' methods is that they pay attention to information flow in an embedded system and its operational environment, and find unexpected obstacles by analyzing deviations of the information flow. Then, they apply the ideas of FTA (Fault Tree Analysis) [10], FMEA (Failure Modes and Effects Analysis) [11], and HAZOP (HAZard and OPerability studies) [12], which are used for the analysis of device failures. This paper describes an Information Flow Diagram (IFD) for conceptualizing information flow in an embedded system and its operational environment. One of the main features of our IFD is that the device diagram and process diagram are combined. This paper also describes a method for analyzing unexpected obstacles using the IFD and the concepts of FTA, FMEA, and HAZOP in an integrated manner. In this research area, individual applications of FTA, which applies a top-down approach, and FMEA and HAZOP, which use a bottom-up approach, have been already studied [13, 14, 15]. Misuse cases [16], abuse frames and goal-oriented obstacle handling [17] have also applied the top-down approach. Integrating the ideas of FTA, FMEA, and HAZOP with IFD is effective for preventing the analysis oversight of unexpected obstacles. In addition, this paper describes experiments using the analysis method, and discusses its results. Section 2 outlines the essentials of the requirement analysis method for unexpected obstacles. Section 3 and 4 describes the IFD and the analysis method for unexpected obstacles, respectively. Section 5 explains our experiments, and Section 6 discusses the results.

\section{Essentials of a Requirement Analysis Method for Unexpected Obstacles}

Before we propose our requirement analysis method for unexpected obstacles in embedded software, we describe the parameters of the method as follows: Business information systems operate using limited kinds of devices such as CPU, memory and hard disk. On the other hand, embedded systems are composed of various kinds of devices such as sensors and controllers. Furthermore, the device types differ according to product. Most unexpected phenomena in embedded systems are caused by exceptional conditions originating from these devices. Moreover, embedded systems are operated not only by users of the systems but also by automatic information triggers such as the light and temperature of the system environment. Exceptional conditions originating in the users and environment also cause unexpected phenomena. Therefore, engineers of embedded systems have to pay attention to users and potential environments as well as devices of the systems. 
When a failure occurs in an embedded system, there must be an originating cause or causes. Moreover, there is a causal relation that proceeds from the originating cause(s) to the failure. The devices in which the causes originate are often different from the device in which the failure is found. The links between the originating cause(s) and the observed failure subsist in the deviation of information flowing between the devices. Therefore, engineers of embedded systems must pay attention to the flow of information and processes which create the flow.

It is not difficult to assume a failure caused by a unique serious exception like a device fault. It is more difficult to assume a failure caused by a set of nonserious exceptions such as overload, deterioration of the device, deviation in the user's operation timing, and environmental influences. The failure can occur when the process receives a set of nonserious deviated information as input, then processes it, outputting seriously deviated information. By investigating the set of nonserious exceptions as failure scenarios, expert engineers of embedded software can anticipate failures which are difficult for novice engineers to anticipate. Therefore, failure scenarios representing a set of nonserious exceptions should be introduced into the requirement analysis method.

Expert engineers of embedded software use the concepts of FMEA, FTA and HAZOP to examine, respectively, the failure patterns of devices, failures occurring contrary to the quality specifications of embedded systems, and deviation of the information that flows in embedded systems. These three points of attention correspond to, respectively, the starting point, the terminal point, and the middle point of a failure scenario. Since the points of attention are frequently scattered in failure scenarios, a systematic approach to scenarios is necessary to avoid oversights of unexpected obstacles. Therefore, FMEA, FTA and HAZOP should be applied together to the analysis method.

\section{Information Flow Diagram}

This section describes the IFD applied to the analysis method of unexpected obstacles. As described in the previous section, it is required for the method that attention should be paid to both devices and processes that are determined by architecture design for implementing expected specifications. Therefore, the IFD is composed of a device diagram (DD) and process diagram (PD). The DD and PD are described in the following subsections:

\subsection{Device Diagram}

Devices and the connections between them are decided upon in the design phase. The devices and connection are illustrated in a DD. Since, as described in the previous section, attention should be paid to users and the environment of the embedded system, we include the users and environment as objects in the DD. For instance, the sun which sends the light and the space through which the light is transmitted are illustrated as devices in the case of street light systems which determine the time of day using optical sensors. 
In the DD, a device is illustrated using a rectangular box, as shown in Figure 1. We divide the rectangular box into three parts, which are used to list the name, attributes and fault patterns of the device, in that order. The fault patterns show the kinds of potential faults such as noise, deterioration and disconnection. The attributes are used to show the properties necessary for the analysis of unexpected obstacles, such as the threshold for noise identification and the time for deterioration.

Information is transmitted between devices by physical media such as electricity, light, and sound. If a fault of the physical media, such as deterioration and disconnection, occurs, the flow of information may be interrupted and a failure may occur. Therefore, it is important in analysing unexpected obstacles to pay attention to physical media that carry information. We call the physical media carrier, and consider that the carrier connects the devices. The DD illustration for a carrier is shown in Figure 2. It uses a diamond box and an arrow. The shaft of the arrow shows the connection between the two devices. The direction of the arrow indicates the direction of information flow. The name of the carrier, its attributes, and fault patterns are described within the diamond box.

\begin{tabular}{|c|c|}
\hline \multirow{2}{|c|}{ Device Name } & \\
\cline { 1 - 2 } & \multirow{2}{*}{ Attributes } \\
\hline Faults & Attributes \\
\hline Fig. 1. Device & Fig. 2. Carrier \\
\hline
\end{tabular}

\subsection{Process Diagram}

We apply the method IDEF0 [18] to statically illustrate the various functions of a process in the PD. In IDEF0, the process is divided into activities. A rectangular box is used to show an activity. The name of the activity is described in the box. If necessary, the input information, output information, control information, and mechanism are specified for the activity using arrows, and their names are given as shown in Figure 3. The activities are connected to each other as part of the information flow. The activity processes input information by the given mechanism, and produces output information. The control information consists of the conditions for the processing.

The PD shows the activities which are processed with the devices shown in the $\mathrm{DD}$, and the information flow between the activities. The relation between the DD and PD is shown by using the connection between the activities and the devices with arrows that indicate the mechanisms. In IDEF0, the process can be described hierarchically in a manner of step-wise refinement. The devices can be also described hierarchically according to subsystems, modules and so on.

The IFD contains not only a representation of an embedded system but also objects for the user and environment of the system. Therefore, the IFD is modelled as a 


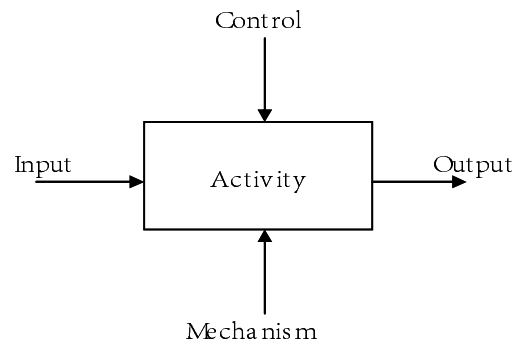

Fig. 3. Process of IDEF0

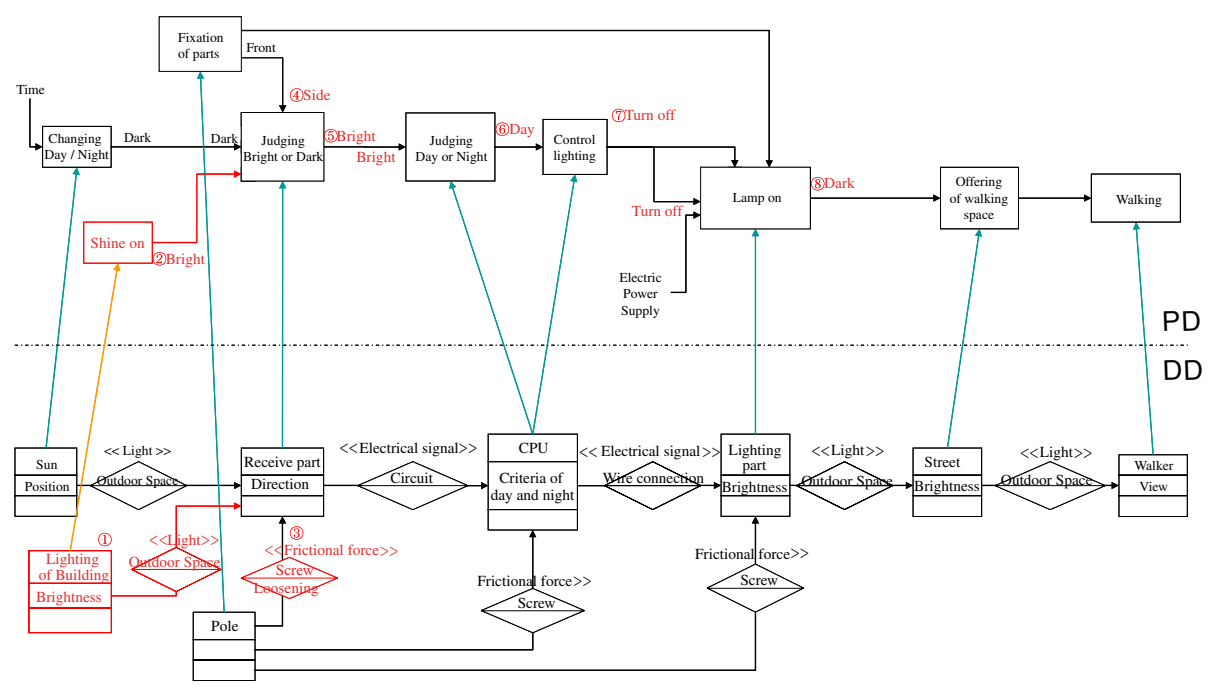

Fig. 4. Example of an information flow diagram

closed world, and information is transferred neither to nor from the IFD. In order to clearly distinguish the information and carrier, we use the term "Information Flow". An example of an IFD is shown in Figure 4.

\section{Requirement Analysis Method of Unexpected Obstacles}

This section describes an analysis method for unexpected obstacles which integrates the concepts of FTA, FMEA, and HAZOP on the IFD.

\subsection{Preconditions of Analysis}

The analysis method is assumed to be applicable after the development phase of an embedded system reaches the following situation. First, the function of the system and the failures against the safety requirements of the system are defined as expected 
Table 1. Examples of Guide-Words

\begin{tabular}{|c|l|}
\hline Type of Influence & Guide-Words \\
\hline Behavior & Stop, Instability, Fixation, $\ldots$ \\
\hline Load & Too much, Too few, $\ldots$ \\
\hline Meaning & Out of range, Undefined, $\ldots$ \\
\hline Procedure & Misorder, Bad timing, $\ldots$ \\
\hline Frequency & Continuous, Repetition, Temporary, ... \\
\hline
\end{tabular}

requirement specifications. Then, there are documents describing the hardware architecture and the faults of the devices in the architecture. In addition, there are documents for the software modules. Thus, after the design for expected requirement specifications is concluded, the analysis of unexpected obstacles begins.

\subsection{Procedure of Analysis}

We show the procedure of the analysis method for unexpected obstacles as follows:

\section{Illustrating the IFD for expected specifications}

Before applying this method, the IFD of the expected specifications is illustrated on the basis of the architectural design of the system.

\section{Additionally illustrating the failure phenomena to be avoided}

The failure phenomena to be avoided are illustrated additionally on the IFD of expected specifications. Specifically, the failure phenomena are related to activity boxes on the PD of the IFD. Serious deviations of the information output from the activity boxes related to the failure scenario are also illustrated on the PD, along with exceptional phenomena that can be assumed by applying guide words to the information flow between any pair of processes on the PD. We show examples of guide words in Table 1.

\section{Analyzing exceptional conditions using the IFD}

We call the conditions for originating exceptional phenomena the exceptional conditions. For each exceptional phenomenon specified in the above-mentioned step (2), its exceptional condition is extracted using the IFD. On the PD, the information flow which has a failure phenomenon is connected to a process which outputs the information. We call this process the "process under examination". The process under examination has its input information, control information, and the device serving as its mechanism. Therefore, the exceptional condition causing the exceptional phenomenon can be classified as follows:

a) Exceptional condition of the device serving as the mechanism of the process under examination. This condition is furthermore classified into the following two conditions:

i. Physical fault of the device. This condition is obtained from the design document of the device.

ii. Logical fault of the device. This condition corresponds to a software bug.

b) Exceptional condition of the input or control information of the process under examination. This condition is furthermore classified into the following three conditions: 
i. Fault of the flow path of the input or control information.

ii. Information received from a disguise device which sends the same kind carrier as the carrier of the input or output information.

iii. Exceptional phenomena of the flow of input or control information.

c) i. combination of two or more of the exceptional conditions described above.

According to the above-mentioned classification, exceptional conditions are searched for the exceptional phenomena under examination. If the exceptional conditions from which the phenomena originate in fact exist, they are adopted. Otherwise, the search for the exceptional conditions causing the phenomena is stopped because the exceptional phenomena have been ruled out. If there is an exceptional condition that is classified into the above-mentioned item b)-iii, the search is repeated for exceptional phenomena newly found from the exceptional condition.

If a disguise device is assumed to appear in the case of the above-mentioned item b)-ii, the disguise device is additionally illustrated. The carrier between the disguise device and the device serving as the mechanism of the process under examination is illustrated. Moreover, a process done on the disguise device is added to the PD. The information flow is also illustrated, from the process on the disguise device to the process under examination.

In this analysis step, the consecutive information flow found in the analysis from an assumed exceptional phenomenon to the fault or disguise device from which the exceptional phenomenon originates is called a failure scenario fragment. It is illustrated on the IFD. Incidentally, it is a top-down approach to first assume an exceptional phenomenon, and then to search for the exceptional conditions from which the phenomenon originates. The search traces the causal relation in reverse.

\section{Constructing a failure scenario}

In the manner of the bottom-up approach which follows the causal relation, a failure scenario originating from the faults found in the previous step (3) is constructed. The detailed procedure is as follows: First, the influence of the faults for output information of the process is assumed. This procedure is repeated according to the consecutive information flow. If the information representing failure is found, the procedure is finished. The consecutive information flow from the faults to the failure constitutes the failure scenario.

In constructing failure scenarios, it is indispensable to take all of the potential combinations of faults into account. However, the combination is made by the confluence of the information flows. Therefore, the failure scenario is pursued by the following procedures:

a) Examine the process that inputs the information under examination. Then, take all combinations of all possible exceptional phenomena of the input information, control information, and mechanism of the process into account. Repeat this procedure if a combination of the possible exceptional phenomena outputs information of an exceptional phenomenon.

b) Finish this procedure if the process can output no information of an exceptional phenomenon. 
c) Reuse the scenario fragment in constructing the failure scenario if the scenario fragment obtained in the above-mentioned step (3) includes the process and its output information under examination mentioned in the previous item a).

This procedure is done for all the faults found in the above-mentioned step (3). The deliverables of the procedure are failure scenarios. In this method, failure scenarios are constructed using the procedure of step (4) after the existence of faults is confirmed using the procedure of step (3). Of course, the other method in which the existence of failures is confirmed prior to faults is also logically applicable. However, expert engineers prefer the method described in this paper.

\section{Experiments}

This section shows a case study of the analysis method described in the previous section, and describes experiments using an IFD of an actual product.

\subsection{Case Study}

We use a street light system with an optical sensor as an example. The specifications of the system are to detect the brightness of the environment with the optical sensor, to judge whether it is day or night, and to turn on or off the fluorescent lamp. The system is illustrated in Figure. 5.

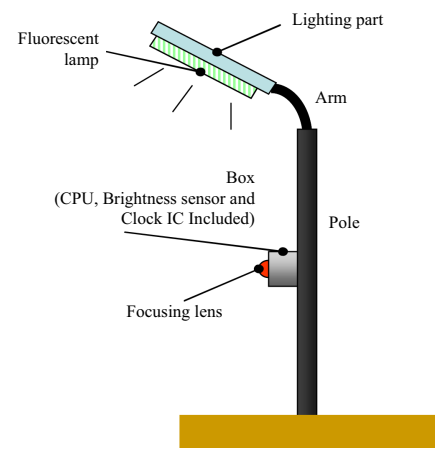

Fig. 5. Street light systems

The obstacles that are necessary to be avoided are shown in Table 2 .

Table 2. Obstacles to be avoided in street light systems

\begin{tabular}{|l|l|l|}
\hline Quality & Obstacle & Carrier \\
\hline \multirow{3}{*}{ Safety } & Burning & Heat \\
\cline { 2 - 3 } & Electrical Shock & Voltage \\
\cline { 2 - 3 } & $\cdots$ & $\cdots$ \\
\hline
\end{tabular}


The procedure of unexpected obstacle analysis for the example is described.

\section{Making an IFD of expected specifications}

The IFD of expected specifications is shown in Figure. 4. In the figure, a failure scenario is also described.

\section{Additionally illustrating failure phenomena to be avoided}

We describe the obstacles shown in Table 2 on the IFD. The result of the examination shows that the lighting parts can burn out. Therefore, the process "Burn out" is added on the PD, and is related to the lighting part via the mechanism as shown in Figure 6. On the other hand, electrical shocks are disregarded since they can not happen in this example.

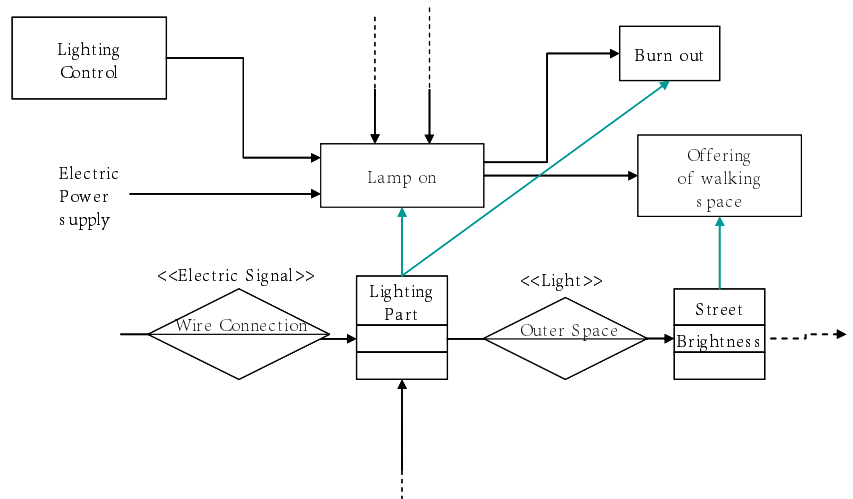

Fig. 6. Addition of process "Burn out"

Moreover, we apply guidewords to each information flow on the PD, and use them to anticipate exceptional phenomena. Examples of exceptional phenomena are shown in Table 3.

Table 3. Example of exceptional phenomena

\begin{tabular}{|c|c|c|l|}
\hline Process & Output information & Guide-Words & \multicolumn{1}{|c|}{ Exceptional phenomena } \\
\hline $\begin{array}{c}\text { Judging } \\
\text { brightness }\end{array}$ & "Bright" & or \\
& "Dark" & Stop & No output information is sent. \\
\cline { 3 - 4 } & & $\begin{array}{l}\text { "Bright" is input; however, the process } \\
\text { output "Dark". }\end{array}$ \\
\cline { 3 - 4 } & Temporary & $\begin{array}{l}\text { The process temporarily outputs } \\
\text { "Bright" during output of "Dark". }\end{array}$ \\
\hline
\end{tabular}

\section{Analyzing exceptional conditions using the IFD}

We analyzed each exceptional phenomenon using a top-down approach, and examined whether exceptional conditions exist for it or not. We show an example of analyzing and examining the exceptional phenomenon in the following: The process "Lamp on" of the mechanism "Lighting part" outputs "Dark" although it is really "Bright". 
a) Assumed fault and exceptional phenomenon

i. Damage, stain, and age deterioration of lighting part

ii. Interception of input information by disconnection of signal line

iii. Interception and decrease in power supply

iv. Exceptional phenomenon of preceding process "Lighting control"

b) Not assumed fault

i. Irregular direction of lighting due to loosened screws

ii. Input information deviation by noise overlay of signal line

The assumed exceptional phenomenon iii is analyzed further, according to the classification of the exceptional conditions described in subsection 4.2.

We assumed the exceptional condition "The light of the building disguises itself as the sun" in the analysis. It represents an exceptional phenomenon that would transmit the information "Bright" although it is really night. In addition, we analyzed exceptional conditions for the control information flow "Directing the optical sensor to the front" output from the process "Fixing devices to pole" whose mechanism is a pole. As the result of this analysis, we can assume the fault "Loosening of the screws that fix the optical sensor to the pole".

\section{Constructing a failure scenario}

We constructed a failure scenario with the bottom-up approach using the fault "Light from a building disguises itself as the sun". Then, on the PD, we traced the flow of information "Bright" output from the process "Lamps on" of the device "Lighting of building". According to the Information flow on the PD, the information reaches the process "Judging brightness". We examine what information will be output from the process by combining the input information, other input information, control information, and exceptional phenomena of the process. In this case, there can be a combination of the input information "Bright" and control information "Directing the optical sensor to the side" because of the fault "Loosening of the screws that fix the optical sensor to the pole". If each of the two pieces of information reaches the process individually, there is no deviation of the output information. However, the combination of the two pieces of information causes the exceptional phenomenon that mistakes "Dark" as "Bright" because the optical sensor receives the light of the building when the loosening of screws causes it to become accidentally turned in the direction of the building. We repeat the procedure for the output information including the exceptional phenomenon mentioned above. Finally, a failure scenario is constructed. We show the scenario in Figure 4 with numbers and comments.

\subsection{Description Experiment of IFD}

In order for a full-scale experimental testing of the analysis method described in this paper, we have to confirm the effective operation organization of the method. Therefore, we have described an experiment on an IFD using an actual product of embedded software. The source codes of the software are about $50 \mathrm{~K}$ steps in size, and are written with $\mathrm{C}$ language. In this experiment, 3 students described the IFD under the guidance of an expert engineer of embedded software.

In this experiment, the students first read the documents of specifications and design. Then, they described the IFD of the expected specifications. The PD and DD 
are divided into 3 layers. The first layer of the DD shows the boundary between the product and its operational environment. The second layer shows the division of the system into the subsystems. The third layer shows the division of subsystems into hardware blocks. Moreover, the 3 layers of the PD are described according to the 3 layers of DD. In describing the IFD of expected specifications, the expert engineer specified only the imperative of describing the 3 layers of the DD. After the students described the IFD individually, we connected the 3 parts of the IFD to each other. Then, a few corrections were made to the IFD. In the next step, the students added the description of failure scenarios which were already found by expert engineers in the product development. In this step, the expert engineers gave the students a simple explanation of the failure scenarios. In this experiment, we found that there were important viewpoints from the device faults and failure phenomena for step-wise refinement of the IFD.

\subsection{Discovering Experiment of Exceptional Phenomena}

Furthermore, we experimented on the use of guidewords by one student to discover exceptional phenomena. In the experiment, the expert engineer first showed 16 guidewords using the format shown in Table 3. The expert engineer gave no further guidance to the student. The experiment included 46 information flows. The student discovered 76 exceptional phenomena in about 7 hours. Most of the exceptional phenomena not discovered by the student were related to the user or the mechanical parts of the system. The examples which are discovered by the student shown in follows:

- Excessive pressure hangs to the operational panel.

- The program beginning instruction is input in spite of while executing the program.

- A mute instruction is input on the way of the control that increases the volume.

The exceptional phenomena which are not discovered by the student shown in follows:

- The interpretation of the instruction displayed on the screen display is mistaken by the user.

- The guidance voice doesn't synchronize with the screen display.

- The machine had stopped before return to the position.

\section{Discussion}

The experiment shown in the previous section has proven the following: By applying the analysis procedure to the IFD of expected specifications under the guidance of an expert engineer, non-expert students could describe the IFD of unexpected obstacle specifications including failure scenarios. Therefore, our IFD-based analytic method can be assumed to be effective as a tool by which a small number of engineering experts in embedded software could guide a large number of novice engineers.

Furthermore, we experimented on the possibility of novice engineers discovering exceptional phenomena using only guidewords. However, this was less successful, especially when it came to errors associated with users and the mechanical parts of 
the system. Therefore, we assume that expert engineers will be required to give more guidance to novice engineers in finding exceptional phenomena.

We have been studying another analysis method applying an analysis matrix other than an IFD [19]. This method constructs failure scenarios by filling in the analysis matrix like a state transition table with exceptional states and events, and by tracing the transitions between exceptional states. This method uses a diagram similar to the DD of the IFD. Of course, the information illustrated in the PD is needed to examine the transitions between exceptional states. In the case of the analysis matrix, however, such information is not explicitly illustrated on the document, but is part of the knowledge base of the expert engineers. Thus, expert skill is required for the method using the analysis matrix. However, the amount of description for the analysis matrix is smaller than that for the PD. Therefore, the method using the analysis matrix is a more efficient method for expert engineers to use than the IFD method.

In the future, we will establish an analysis method using IFD by experimenting with it in real applications. Then, we will integrate the two methods. Furthermore, we will study the effective use of the integrated method by a group composed of a small number of expert engineers and a much larger number of novice engineers. When integrating the methods, we will formalize the integrated method with the qualitative reasoning theory that applies the notion of state transition and constraint conditions. By formalizing the method, we will formalize the experts' knowledge of unexpected obstacles. Then, we will develop a knowledge base according to the formalization of knowledge. The knowledge base will be installed in the CASE tools for analyzing unexpected obstacles. Incidentally, the IFD has the characteristics of a directed graph. Therefore, we will also study the application of the graph method. It will be useful for finding exceptional phenomena such as loss of information flow because of disconnection of a cable, occurrence of new information flow because of electromagnetic induction, and positive or negative feedback on the information flow loop.

\section{Conclusion}

This paper has described an IFD and an analysis method based on the IFD for analyzing unexpected obstacles in embedded systems. The IFD illustrates the users, environment, and devices in addition to the processes of the systems, all of which sometimes cause unexpected obstacles in embedded systems. In order to analyze unexpected obstacles using both top-down and bottom-up approaches for preventing omissions in analysis, we have jointly applied the concepts of FTA, FMEA and HAZOP. We have confirmed that novice engineers can describe the IFD of expected specifications and the IFD of unexpected obstacle specifications under the guidance of expert engineers in an experiment applying the method to an actual product.

In the future, we will establish the analysis method after further experiments using the IFD-based analysis method. Then, we will integrate the IFD and analysis-matrixbased analysis methods. After that, we will study the quality and efficiency of the new analysis method by considering the possible structure of engineering personnel in a firm using the method. Moreover, we will study the knowledge base of unexpected obstacles and a graph analysis method to use with the IFD. 
Acknowledgement. The authors would like to thank Mr.Tanabe, Mr.Tanimoto, Mr.Inoue, and Mr.Kubo for cooperation in the experiment.

\section{References}

1. Mise, T., Shinyashiki, Y., Hashimoto, M., Ubayashi, N., Nakatani, T.: A Specification Analysis Method for Unexpected Obstacles in Embedded Software (in Japanese). Proc. of the FOSE2005, Kindai Kagaku Sha. Japan Society for Software Science and Technology, pp. 227-235 (2005)

2. Sumi, T., Hirayama, M., Ubayashi, N.: Analysis of the external environment for embedded systems, IPSJ SIG Technical Reports, 2004-SE-146, pp. 33-40 (2004) (in Japanese)

3. Ministry of Economy, Trade and Industry, editor. Report of actual field survey of embedded software, Edition, Ministry of Economy, Trade and Industry (2005) (in Japanese)

4. Crook, R., Lnce, D., Lin, L., Nuseibeh, B.: Security Requirements Engineering: When Anti-Requirements Hit the Fan. Proc. of the 10th Anniversary Joint IEEE International Requirements Engineering Conference (RE’02), pp. 203-205 (2002)

5. Hatanaka, H., Shinyashiki, Y., Mise, T., Kametani, H., Hashimoto, M., Ubayashi, N., Katamine, K., Nakatani, T.: An Analysis of Information Flow Graph based on Conceptual Model of Exceptions in Embedded Software, Technical Report of IEICE 104-431, pp. 19-24 (2004)

6. Shinyashiki, Y., Mise, T., Eura, Y., Hatanaka, H., Hashimoto, M., Ubayashi, N., Katamine, K., Nakatani, T.: A Conceptual Model of Exceptions in Embedded Software. In: Proceedings of Embedded Software Symposium, pp. 8-11 (2004) (in Japanese)

7. Mise, T., Shinyashiki, Y., Eura, Y., Hatanaka, H., Hashimoto, M., Ubayashi, N., Katamine, K., Nakatani, T.: Exception Analysis Matrix for Embedded System Software Specification. In: Proc. IPSJ/SIGSE Embedded Software Symposium (ESS 2004) (in Japanese) (2004)

8. Mise, T., Shinyashiki, Y., Hashimoto, M., Ubayashi, N., Katamine, K., Nakatani, T.: An Analysis Method with Failure Scenario Matrix for Specifying Unexpected Obstacles in Embedded System. The proceeding of the 12TH Asia-Pacific Software Engineering Conference, pp. 447-454 (2005)

9. Kametani, H., Shinyashiki, Y., Mise, T., Hashimoto, M., Ubayashi, N., Katamine, K., Nakatani, T.: Information Flow Diagram and Analysis Method for Unexpected Obstacle Specification of Embedded Software. Proc. of the Knowledge-Based Software Engineering (JCKBSE'06), pp. 115-124 (2006)

10. Leveson, N.G.: Fault Tree Analysis, Safeware System Safety and Computers, pp. 317 326. Addison-Wesley, Reading, MA (1995)

11. Leveson, N.G.: Failure Modes and Effects Analysis, Safeware System Safety and Computers, pp. 341-344. Addison-Wesley, Reading, MA (1995)

12. Leveson, N.G.: HaZards and Operability Analysis, Safeware System Safety and Computers, pp. 335-341. Addison-Wesley, Reading, MA (1995)

13. Pentti, H., Atte, H.: Failure Mode and Effects Analysis of Software-based Automation Systems, STUK-YTO-TR 190, p. 35 (2002)

14. Dehlinger, J., Lutz, R. R (eds.): Software Fault Tree Analysis for Product Lines. Proceedings of the Eighth IEEE International Symposium on High Assurance Systems Engineering, pp. 12-21 (2004) 
15. Redmill, F., Chudleigh, M., Catmur, J.: System Safety: Hazop and Software Hazop, p. 248. John Wiley \& Sons Ltd, New York (1999)

16. Alexander, I.: Misuse cases, use cases with hositile intent. IEEE Software 20(1), 55-66 (2003)

17. Lamsweerde, A.V., Letier, E.: Handling Obstacles in Goal-Oriented Requirements Engineering. IEEE Transactions on Software Engineering 26(10), 978-1005 (2000)

18. Bemus, P., Mertins, K., Schmidt, G. (eds.): Handbook on Architectures of Information Systems. Springer, Heidelberg (1998)

19. Mise, T., Hashimoto, M., Katamine, K., Shinyashiki, Y., Ubayashi, N., Nakatani, T.: A Method for Extracting Unexpected Scenarios of Embedded Systems. Proc. of the Knowledge-Based Software Engineering (JCKBSE'06), pp. 41-50 (2006) 